\title{
Le récit de soi et la production de la parole politique de la victime dans les posts sur les réseaux sociaux numériques
}

\author{
Angie Biondi \& Angela Cristina Salgueiro Marques \\ Universidade Tuiuti do Paraná, Brasil / UFMG, Brasil \\ E-mail : angiebiondina@gmail.com/angelasalgueiro@gmail.com
}

\begin{abstract}
Résumé
L'objectif de cet article est d'étudier les manières langagières de quelques exemplaires récents publiés dont des femmes victimes d'agression sexuelle éla- sur les réseaux sociaux numériques. Ces récits réborent, sur des réseaux sociaux comme le Facebook, vèlent un certain travail éthique de construction subleurs posts en tant qu'une énonciation de «récits jective de la victime dans l'expérience d'écriture et de soi» (Butler, 2015). Le parcours méthodologique d'enregistrement en ligne, ce qui permet au sujet vise analyser la façon dont ces récits convoquent une d'examiner de façon critique son statut par rapport adhésion aux expériences de douleur et souffrance aux discours normalisateurs, en quête d'affirmation qui traversent différents domaines d'affinité et qui de nouveaux moyens d'expression subjective, polifigurent comme appel à la responsabilité éthique. tique et sociale.
\end{abstract}

Notre effort analytique privilégie les matérialités

Mots-clés : énonciation; récit de soi ; victime; réseaux sociaux numériques; politique.

\section{The account of oneself and the creation of a victim's political talk on testimonial posts in networks ${ }^{1}$}

\begin{abstract}
In this text we search to observe how the victims articulate their posts in the enunciative form of the "account of oneself" (Butler, 2015), in order to convoke adhesion and empathy to their experiences of pain and suffering generated by a varied affinity fields. Our aim is to show that the practices of giving an account of oneself are also a call to an ethical responsibility. The analysis effort privileges the language

materialities of some recent public posts in the networks. The testimonies here analyzed disclose a certain ethical work of subjective construction of the victim in the experience of writing and register online, which allows the individual to critically examine its condition in relation to normative speeches, in search of the affirmation of new ways of subjective, social and political expression.
\end{abstract}

Keywords : enunciation; testimony; victim; social network; politics.

Data de submissão : 2017-12-30. Data de aprovação : 2018-04-12.

A Revista Estudos em Comunicação é financiada por Fundos FEDER através do Programa Operacional Factores de Competitividade - COMPETE e por Fundos Nacionais através da FCT - Fundação para a Ciência e a Tecnologia no âmbito do projeto Comunicação, Filosofia e Humanidades (LabCom.IFP) UID/CCI/00661/2013.
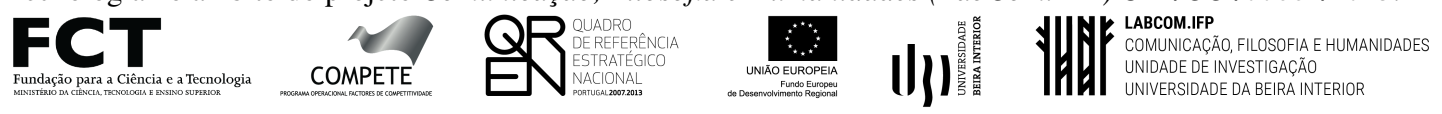

1. Cet article fait parti des résultats partiels de la recherche "Solidarité en réseau : la visibilité et les expériences de victimes dans la culture contemporaine", financée par le CNPq. 


\section{INTRODUCTION}

CET article présent deux aspects principaux du processus de construction de la victime dans les récits de soi élaborés et diffusés par les échanges numériques en ligne : le premier vise à poser une problématique sur la légitimité de la parole revendiquée par la victime, à partir de la déclaration et de l'exposition de leurs expériences de douleur et de souffrance quotidiennes qui sont aujourd'hui produites et partagées sur le réseau. Le second vise à déterminer comment les formes énonciatives, en tant que «récits de soi» (Butler, 2015), observées dans les récits-posts analysés, gèrent des champs d'affinité variés et des interactions affectives selon leurs histoires et leurs causes.

Notre grille de recherche qualitative est fondée sur l'approche de "l'ethnographie virtuelle" (Hine, 2004 ; 2015 ; Recuero, 2013) associée à ce qu'on appelle "les méthodes d'analyse biographique et autobiographique" (Barros; Silva, 2002; Goldenberg, 2004). La référence commune entre les méthodes dits autobiographiques est la dimension narrative, le fait de raconter une histoire (Barros ; Silva, 2002 : 136.), ce qui est au cœur de la méthode elle même.

C'est pour cela que nous prenons les récits des victimes tels qu'ils sont afin de préserver leur dimension matérielle et narrative et d'observer les questions suivantes : a) la manière descriptive utilisée par le sujet lui-même pour raconter son histoire; b) l'organisation séquentielle des événements ; c) l'intention sous-jacente ou exposée dans le récit-post; d) l'interpellation du lecteur afin de comprendre, même à titre préliminaire, comment la victime s' énonce, en créant et en partageant son expérience vécue de la douleur et de la souffrance à travers les réseaux sociaux et de relations.

Nous croyons que l'émergence de ces nouvelles formes d'énonciation qui traversent, entremêlent et reconfigurent l'énonciation de la victime tel qu'on décrit dans cette étude, allié à la méthode autobiographique, indique une certaine autonomie des processus qui émergent et prolifèrent dans les réseaux et expose également des changements dans la manière d'établir des liens et des interactions, proprement affectifs, qui se développent entre les sujets et leurs causes.

Selon Christine Hine (2004), il est important de considérer l'Internet comme un élément ou un artefact culturel, une fois que plusieurs espaces d'interaction s'y constituent à partir de l'agencement de vraies personnes avec des objectifs et des priorités situées et définies dans un certain contexte, façonnés par la manière dont les réseaux, ainsi que ces dispositifs, ont été commercialisés, enseignés et utilisés. C'est à dire que nous devons tenir en compte sa dimension pratique, concrète et quotidienne. Nous considérons que le regard qu'accompagne et observe les usages, les appropriations et les trajectoires des individus dans ce champ - poreux et flexible - permet une compréhension de ce qui est efficace dans les enjeux quotidiens placés sur les réseaux sociaux en ligne.

Les phénomènes observés dans les lieux d'échange communicationnel sur le réseau numérique doivent être considérés comme étant pénétrés par les actions du quotidien et incorporés au pratiques des sujets (Hine, 2015 : 170). Par conséquent, il s'agit d'une méthode appropriée pour définir un champ d'observation et de collecte de données. En effet, cette méthode respecte la densité descriptive et interprétative en cause, considérant à la fois le phénomène et la recherche, mais surtout parce qu'elle met l'accent sur la dimension complexe du traitement et de la comparaison 
des informations et de l'analyse. Le modèle construit selon l'approche et la méthodologie choisis se révèle adéquat, pertinent et productif puisqu'il valorise des différences descriptives et la problématisation des dimensions interlocutives qui organisent et soutiennent les échanges en ligne. Bref, il s'agit d'un approche qui met en relief les usages et les appropriations de formes diverses d'énoncés et d'engagements du chercheur avec les donnés.

En ce cas, aller sur le terrain ne signifie pas réverbérer une superposition entre la logique commerciale ou institutionnelle des moyens ou dispositifs techniques et la production des énoncés par les sujets, mais bien les observer comparativement. Nous faisons référence ici à une sorte d'enquête qui cherche à observer, documenter, interroger et analyser les données en tenant en compte qu'ils sont toujours ouverts et mouvants et que le processus descriptif-interprétatif est permanent et actif.

Selon Goldenberg (2004), il faut observer, dans les trajectoires singulières, les reflets des conditions historiques et culturelles dans lesquelles elles se trouvent. Les approches ethnographiques avec leurs méthodes de recherche qualitative rendent possible l'approximation entre les expériences réelles des individus et leurs formes expressives, tout en considérant les articulations productives entre deux dimensions centrales : la vie et l'écriture.

La recherche réalisée nous a permis, par l'approche qualitative de l'ethnographie virtuelle, de créer un type spécial de document d'analyse dans laquelle l'expérience personnelle s'entremêle à la compréhension active d'un contexte social et historique plus large, dans lequel elles se mélangent en atténuant ainsi les antagonismes canoniques qui délimitent les distinctions entre la subjectivité d'un récit individuel et l'objectivité de la vie sociale.

Cette étude a privilégié un type de méthode capable d'empêcher les données de perdre leur caractère unique et à la fois caractéristique d'une ambiance communicationnelle qui opère dans une autre logique espace temporelle. Il est important de noter que nous n'avons pas eu la prétention d'analyser les échantillons en tant que représentation universelle du phénomène d'agression aux femmes, car il est impossible d'évoquer la violence dans une dimension généralisée en pérennant uniquement des récits-posts de quelques victimes.

Bien au contraire, nous avons cherché à développer une attention particulière aux nuances qui se sont présentes dans les données recueillis sur le terrain et qui sont des formes expressives significatives d'une expérience unique de la douleur et de la souffrance. En outre, nous avons cherché à établir des parallèles et des associations avec des aspects socioculturels et politiques propres à notre contexte, pour comprendre, par l'artifice de la comparaison, comment les articulations s'y font. Il s'agit de consacrer plus d'attention aux données afin de comprendre comment le sujet, par son propre discours à lui - le récit de soi ici entendu comme un genre d'énonciation qui vise, avant tout, le partage - organise son expérience vécue, en le rapportant à travers les ressources technologiques et communicationnelles de plus en plus ordinaires au quotidien telles que les réseaux sociaux.

Notre recherche a mené la collecte et la sélection des données empiriques, qui ne se détachaient pas de son contexte de partage, parallèlement à l'étude des cadres théoriques qui encouragent les formes d'énonciation de la victime comme objet d'étude. Comme résultat nous avons réunis environ une vingtaine de récits, capturés sur les deux réseaux sociaux les plus importants (Facebook et Instagram), depuis février 2015. 
Nous avons élaboré un carnet de récits en tant qu'outil de caractère descriptif, pour la collecte, l'accompagnement et l'analyse préalable. Il nous a permit d'organiser les informations provenant de l'échantillon, en réservant également un espace au chercheur, dans lequel il pouvait élaborer des commentaires sur les expressions, les mots et les thèmes récurrents qui ressortaient de l'observation conjointe. L'examen de l'intersection de ces aspects descriptifs et analytiques des données, met en relief les récits-posts des victimes, en associant leurs gestes d'énonciation à des modes de partage disponibles sur les réseaux sociaux, ce qui indique la façon dont ces récits de soi interpellent et créent identification et adhérence.

Goldenberg fait noter que «l'utilisation de la méthode biographique en sciences sociales est une manière de révéler comment les gens universalisent, à travers leurs vies et leurs actions, le temps historique dans lequel ils vivent» (2004, p. 43). Toute forme biographique est toujours un portrait de conversations et aussi de négociations.

\section{“YOU WILl NOT SILENCE ME" : LA PAROLE REVENDIQUÉE PAR LE SUJET}

Le 6 Janvier 2016, Amber Amour, une femme de 27 ans, a publiée sur sa page Instagram le récit de l'agression sexuelle qu'elle avait souffert quelques minutes avant d'écriture le message. Militante des Droits de l'Homme, elle était à Cape Town, Afrique du Sud, pour promouvoir la campagne «Stop rape : Educate », qui visait à informer la population locale à propos de la violence sexiste et à soutenir les femmes victimes d'agressions.

La jeune a racontée qu'elle séjournait dans une auberge avec un groupe de collègues quand elle a trouvé un garçon qui l'avait embrassé une fois. Dans la conversation, elle dit que le jeune homme l'a offert la salle de bain de chez lui, parce que l'auberge avait que de l'eau froide et qu'elle était malade depuis deux jours :

J'ai accepté, parce que l'eau de l'auberge où j'étais logée était très froide et, il y a deux jours, j'étais très malade [avec une intoxication alimentaire]. Je voulais juste une douche chaude. Dès que je suis rentrée dans la salle de bain, il a forcé mes genoux. Je lui ai demandée d'arrêter. Mais il est devenu plus violent. Il m'a élevé et il m'a pénétré. Je lui ai demandé d'arrêter à nouveau et j'ai commencé à pleurer. Quand il a poussé mon cul, je me suis évanouie. Je me suis réveillé quelques minutes plus tard et je l'ai vu essayer de ramper hors de la porte. Quand il m’a vu éveillé, il est revenu me déchirer sous la douche ${ }^{2}$.

Elle a également publiée deux photos : une sur le chemin vers l'hôpital, et un autre encore au lit, en attendant la réalisation de l'examen qui établi la preuve de la violence. Dans l'image, il est possible de voir une partie de ces jambes recouvertes par le drap de l'hôpital, de haut en bas, comme prise par une perspective à la première personne. La visualisation des jambes dans une position qui est équivalente au regard de la jeune sur les membres inférieurs de son corps, est présentée ironiquement par Amber : « Ma vision du kit de viol », dit-elle.

2. Tout les extraits du récit cités dans cette étude ont été traduits pour nous et sont disponibles dans la page d'accès gratuit, publié le 21 Novembre, 2015 Amber Amour. Disponible en www.instagram.com/ambertheactivist. Accès au 06.01.2016. 
Quelques jours plus tard, l'Instagram a supprimé le message et les photos d'Amber. Selon la plate-forme, les règles de la communauté Instagram interdisent le partage de contenu considéré comme inapproprié. Le même jour, la jeune fille a affiché un bref message exigeant une réponse immédiate d'Instagram sur la disparition de son récit :

Excusez-moi, Instagram, où est parti mon post? Le post juste après le viol est disparu et il y avait ce message d'IG quand je suis entrée ce matin. Qu'est-ce qui ce passe, putain? Depuis quand est-il contre les règles de la communauté se montrer soi-même? Je voudrais une explication, mise à jour, de l' @instagram. Vous n'allez pas me taire. Vous avez supprimé de ma page le post documentant le viol, mais devinez quoi ? Cette merde avait déjà viralisé. Elle est partout sur Internet, alors tout ce qui est derrière l'exclusion, en fait, ça sert à rien ! Les survivantes de viol sont appelées des menteuses et des fraudes à chaque jour! Je trouve absolument scandaleux que le post a été supprimée. Je ne serai pas réduite au silence. Je vais continuer à dire ma vérité.

Cependant, avec plus de 20.000 abonnés à ce jour, Amber, elle-même, a annoncé que son post avait viralisé et qu'il a été vu des milliers de fois. Ensuite, l'Instagram a présenté ses excuses en public et il a libéré l'affichage du post et a également souligné le courage de l'initiative de la jeune de partager son expérience et d'offrir l'occasion d'une discussion collective sur une question aussi importante.

Il a fallu beaucoup de courage à Amber pour partager son histoire, et nous voulons la diriger nos profondes excuses. Nous avons fait une erreur et le contenu est déjà publié. Nous voulons toujours que notre communauté se sent en sécurité et soutenue, et qu'elle soit en mesure de discuter de questions et des expériences avec les autres.

Nous savons que les ressources technologiques qui élargissent les possibilités de production et de partage de contenu deviennent à la fois des instruments potentiels de surveillance et de contrôle, "les dynamiques de surveillance sur Internet sont désormais étroitement liés aux formes de participation des utilisateurs et des affrontements auxquels ils se rapportent "(Bruno, 2013, p. 125). La suppression du récit et des photos d'Amber est juste un des nombreux exemples parmi les pratiques de surveillance menées par les gestionnaires de réseaux sociaux eux-mêmes sur tout le matériel produit et partagé par ses utilisateurs. Dans ce contexte, nous savons que les intersections entre l'énonciation et la visibilité sont entremêlées aux pratiques de surveillance et de contrôle qui déplacent les processus de la sociabilité contemporaine.

Sur ce point, nous voulons mettre en évidence la façon dont la demande d'Amber est devenue de plus en plus fréquente chez les utilisateurs du réseau numérique qui comprennent et réclament, aujourd'hui, pour eux-mêmes le droit et la légitimité de proclamer leurs propres causes, car ils sont les responsables directs pour générer et mettre à la disposition volontairement, leurs histoires de vie et leurs expériences quotidiennes.

La tension entre l'énonciation libre, actuellement considérée comme un droit du sujet ordinaire, et la visibilité encore normalisée de certaines pratiques de communication - comme celles qui apparaissent sur les réseaux sociaux - acquiert des contours plus intenses lorsque nous explorons un peu plus de questions concernant l'expérience narrativisée de l'intimité (inscrite ici par 
la souffrance et la violence) d'un sujet, comprenant le regard de l'autre, a partir des dispositifs technologiques.

La production d'une subjectivité dans laquelle le soi est guidé par le regard d'autrui semble devenir un consensus théorique de nombreuses études de communication aujourd'hui (Bruno, 2013; Sibilia, 2008). Les pratiques contemporaines d'énonciation du sujet, dont les réseaux sociaux et des relations y font partie, mettent en évidence le rôle central assumé par l'affichage, l'apparence et l'image comme une démarcation socioculturelle prédominante pour la consolidation d'un moisujet :

Si les dispositifs modernes de visibilité creusaient une subjectivité intériorisée qui, a partir du regard de l'autre, établissait l'auto-surveillance, aujourd'hui, il semble d'être en formation, une subjectivité extériorisée, où la prise en charge du contrôle et du soin de soi-même se fait par l'exposition publique, à portée du regard, de l'examen minutieux ou de la connaissance de l'autre. Le déterminant ici est de comprendre cette subjectivité qui se module comme extériorité, dans le mouvement même de se rendre visible à l'autre (Bruno, 2013, p. 67).

Selon ce point de vue, les aspects constitutifs et légitimateurs dans le récit d'un moi-sujet, comme l'authenticité et la vérité, seraient encore participants à ce processus, bien qu'également modulés par la valeur de l'affichage. Se présenter, se faire image, se raconter, il est aussi se constituer en tant que sujet, et dans une large mesure, garantir sa place de parole. Ainsi, dans la mise en place des actions telles que voir / être vu, raconter/ se raconter, «dans les pratiques en question, la place où le soi se réalise et devient efficace est proche du regard de l'autre, dans sa capacité d'être vu, et non pas autant par la rétraction d'une intériorité opaque» (Bruno, 2013, p. 70). Cela explique l'exigence d'Amber de se montrer soutenue par la mise en valeur de la vérité que constitue son récit. Son droit à la parole ne peut être dissocié de son lieu de visibilité par l'image, car les deux sont constitués et légitimés par le regard d'autrui.

Tel droit à la parole et à l'image est présent de plus en plus dans la revendication des sujets communs aux expériences qu'ils partagent. "Je vais continuer à dire ma vérité », proclame Amber, dont la voix semble multipliée par les formes extensives et diverses de partage, de likes et de visualisations ultérieures. Dans la production de la subjectivité contemporaine, l'authentique et le vraie d'une expérience narrativisée ne sont plus confinés à la dimension intime en tant qu'intériorité, au sens d'une préservation radicale et d'une protection individuelle. Au contraire, ce genre d'expérience semble s'affirmer par la valeur d'présentation et d'exposition. Ainsi, ce renversement renforcerait une attitude révélatrice qui devient encore plus puissante par les formes d'énonciation possibles au sujet aujourd'hui. En effet, il densifie le caractère authentique et vrai de l'expérience en soulignant sa valeur dans les formes de présentation immédiates et directes.

Dans le récit-post d'Amber, la jeune fille déclare «sa vérité» et se justifie quand elle compare la possibilité de cacher ses souffrances et de sauvegarder son expérience intime de la douleur et la possibilité de révéler la vérité à d'innombrables inconnus. Ainsi, sa vérité à elle et la vérité de la situation sont équivalentes à la dimension énonciative et visuelle, mais, surtout, morale (Fassin, 2011). L'expérience narrativisée de la souffrance (chez Amber) présente un caractère très fort de revendication qui ne permet pas la suspicion ou la suspension de la parole de la victime. Une question importante serait de savoir comment les individus se gouvernent, eux-mêmes et parmi eux, 
par la production sociale de leurs vérités, disait Foucault (2004). «La victime et son expérience traumatique désignent une réalité irréfutable associée à un sentiment d'empathie qui imprègne chaque espace moral des sociétés contemporaines" (Fassin 2011, p. 17).

Merci à vous tous de votre soutien pendant cette période. Vos messages m'ont encouragés à prendre l'initiative et à me mettre debout pour moi et pour toutes les survivantes de viol. Pour ceux qui préfèrent me culpabiliser ou toute autre survivante quiconque, je veux que vous sachiez que vous êtes la raison pour laquelle je suis brutalement franche. Je pourrais avoir caché les détails, garder quelques informations seulement pour moi, mais non. Vous avez besoin de connaître la vérité et voir la réalité de la situation. Peu importe ce que la personne fait, rien n'est une invitation au viol. Peu importe si je l'ai embrassé. Peu importe s'il était ivre. Peu importe si j'ai dit «oui» à un bain. Je n'ai jamais dit qu'il pouvait être violent avec moi. Je n'ai jamais dit qu'il pouvait me faire du mal. Je n'ai jamais dit qu'il pouvait me violer.

Sa déclaration indique également qu'il existe une force positive marquée par l'utilisation emphatique de sa volonté répétée dans les opposés peu importe ce qu'il versus j'ai jamais dit. L'expression positive et revendicative d'un moi-sujet qui peut et doit raconter son expérience est la voie de légitimation de toute son action ou attitude. Si vous ne pouvez pas soumettre une à l'autre, il ne sera également pas possible de soumettre la parole de l'un à l'autre. Ainsi, le fait de forcer le silence d'Amber dans ce contexte est configuré comme une autre agression, un autre type de violence par la soumission de la parole et du sujet. Ici, l'interdit de la confession et le secret de la douleur intérieure ne pourrait vraiment pas être restreint, puisque tout investissement d'énonciation de sa douleur apparaît déjà marqué par l'image : cet investissement est orienté vers l'extérieur et cherche sa réalisation par le devenir collectif de la narrative, c'est à dire, chez autrui :

Cependant, nous pouvons penser à l'émergence d'autres domaines d'investissement qui sont configurés selon une autre topologie : l'intériorité, bien qu'elle y ait encore présente, elle cesse d'être le principal objectif des soins et de contrôle, et, ne peut plus être la demeure même de la vérité ou du désir. Si la modernité a produit une topologie de la subjectivité et du quotidien qui circonscrivait l'espace privé et ses différents niveaux de vie intérieure - la maison, la famille, l'intimité, le psychisme -, l'actualité renverse cette topologie et met la subjectivité à l'espace ouvert des médias et ses différents niveaux de vie extérieure - l'écran, l'image, l'interface, l'interactivité. (Bruno, 2013, p. 81).

L'enregistrement immédiat et son partage jouent un rôle important dans le processus de constitution de la subjectivité extériorisée, non seulement pour permettre la publicité des événements de la vie des sujets ordinaires, mais parce qu'il acquiert également un rôle moral dans la légitimité de ce qui parle et s'expose. Il s'agit de la victime elle-même qui se présente et revendique l'image et la parole, car elle même incarne l'instance morale et politique légitime de ses douleurs et expériences uniques. Il y a une construction de la place de la parole du sujet qui conscient de son statut et de la projection qu'il peut atteindre, se présente comme un agent qualifié de la douleur réelle et légitime qu'il éprouve. En autres termes, il est porteur authentique d'une expérience originaire capable de montrer la vérité des choses qui méritent d'être partagées : «la construction de la personne comme victime dans le monde contemporain est considérée comme un moyen de 
donner une sorte de reconnaissance sociale à la souffrance, en la circonscrivant et en lui donnant de l'intelligibilité» (Sarti, 2011, p. 54).

Ainsi, nous dirions que le partage devient une action complémentaire et extensive à la situation de douleur et de souffrance dans laquelle se trouve le sujet qui la vit et la raconte au même temps ; par conséquent, la victime prend simultanément les rôles de narrateur et de personnage.

En outre, il faut noter que la plainte devient un impératif à la victime que en dehors d'exprimer sa douleur, elle est contrainte à la confession. La responsabilité de l'expérience partagée se tombe sur elle. Au nom de nombreuses autres victimes, réelles ou potentielles, elle ne peut pas se taire. Il faut s'exprimer : que ce soit entre des cris ou sanglots.

Dans cette perspective, les pratiques d'exposition de soi rendues possibles par les nouvelles technologies de communication n' acquérir cette pertinence significative que dans le réagencement de la douleur et de la souffrance entre les sphères publique et privée, de la modestie de la douleur intérieure à l'exposition de la plainte. Il s'agit d'une sorte de politique du regard que s'établisse, de plus en plus engagé à la valeur d'exposition gérée par les différentes ressources technologiques de production et de partage de contenu disponibles aujourd'hui.

\section{LE RÉCit de SOI DANS LA PRODUCTION DE LA PAROLE POLITIQUE DE LA ViCTIME}

«Je me suis rasée la tête parce que je suis fatiguée d'être harcelée. Je ne me tais plus».

Un autre aspect qui semble donner d'ampleur à la réflexion de ce problème est la quête d'approfondir la perspective qui explore le lieu d'énonciation par la densification (le renforcement) de la notion du moi-sujet intégrée dans la conception du récit comme expression privilégiée de la visibilité de la victime. L'écriture du soi, se raconter, «la construction du récit de soi» (Butler, 2015) sont impliqués dans la construction de la subjectivité qui maintient son ouverture et la nature procédurale de l'être en tant que devenir par l'autrui.

Il est important de se rappeler que le récit de soi se développe dans une scène d'interpellation dans laquelle, selon Butler (2015), se combine une sorte d'éthique de la responsabilité avec la véracité du récit, et également avec le type de liaison qu'il peut établir avec les interlocuteurs. Le récit est toujours une action ciblée à la fois à la l'autorévélation, à l'auto-transformation et à la configuration des conditions et des cadres de sens discursifs qui définissent ce qui parle. «Quand nous agissons et parlons, non seulement nous nous révélons, mais aussi nous agissons sous les régimes d'intelligibilité qui déterminent qui sera l'être qui parle, en les soumettant à une rupture ou à l'examen, en consolidant ses normes ou en contestant son hégémonie» (Butler, 2015, p. 167).

Malgré son déclenchement par une accusation violente, le récit de soi entrepris par Amber ne se confond pas avec la confession qui implique un individu coupable, pêcheur, qui se méfie de soimême et doit trouver les erreurs et les écarts de son caractère pour justifier la violence sexuelle, afin de se corriger et de s'adapter aux normes établies et au régime consensuel dominant. Bien au contraire, son récit révèle un travail éthique de construction subjective dans l'expérience dissensuelle de l'écriture et de l'inscription en ligne. Cela permet à l'individu d'examiner de manière critique son statut par rapport aux discours normalisateurs, en quête d'affirmation de nouveaux moyens d'expression subjective, politique et social (Rago, 2013). 
Dans un autre cas que nous avons analysé, l'étudiante chercheuse Thais Moya a publié sur sa page Facebook, un récit, sous la forme de protestation, qui raconte l'harcèlement sexuel qu'elle a subie de la part de son directeur de thèse. Selon elle, le cas avait déjà été informé au bureau de son Programme d'études supérieures. Cependant, il a été ignoré. Alors que l'harcèlement a continué, Thais a décidé d'utiliser sa page sur les réseaux sociaux pour «donner la parole» au cas et raconter la vérité des faits.

Le 11 Décembre 2014, la jeune fille a affiché un selfie avec la tête rasée suivi du récit de sa douleur en protestant :

Je me suis rasée la tête en signe de protestation. Je me suis rasée les cheveux parce que j'étais deux fois attrapée et embrassée par mon professeur et (ancien) directeur de thèse sans mon consentement. Je me suis rasée la tête parce que, comme presque toutes les victimes d'harcèlement, j'ai passé deux ans effrayée et sous la contrainte par les relations de pouvoir qui sous-tendent les conséquences de le dénoncer.

Je me sentais responsable non seulement pour ma carrière professionnelle, mais aussi pour celle de l'enseignant concerné et les conséquences négatives contre mon Programme d'études supérieures et contre mes collègues du centre d'études. Je suis restée silencieuse, intimidée. Je me suis rasée les cheveux parce que, après avoir été saisie par l'enseignant et lui rejeter, il m'a banni peu à peu des projets du centre d'études sous sa coordination, en disant une fois que je suis «une poulette sans doctorat, qui a besoin de mettre la queue entre les jambes, d'arrêter de confronter l'enseignant docteur et d'apprendre à jouer le jeu de l'académie, si je veux y continuer". [...]

Je me suis rasée les cheveux parce qu'un groupe d'étudiants ont lu les récits d'harcèlement et se sont mobilisés, en écrivant une lettre aux enseignants (CPG), revendiquant leur prise de position, principalement parce qu'ils avait complètement ignoré les récits. Une grande partie des enseignants est devenu très en colère. Ils ont commencé à mettre de la pression sur ses doctorants pour démobiliser la revendication en cours.

Je me suis rasée la tête parce que la coordinatrice du Programme a publié sur sa page Facebook que les étudiants étaient «en train de criminaliser l'expression sensuelle». Je me suis rasée les cheveux car hier, il y avait un rendez-vous fixé par enseignants avec étudiants. Sans aucun scrupule, l'enseignante, en parlant au nom de la faculté, a donné les noms indiqués sur les récits, jusque-là anonymes, et a rajouté - fondée sur des arguments qui font appel à la tradition - que mes récits sont du mensonge, car elle connaît l'enseignant depuis 20 ans et, selon elle, il est un homme engagé et galant (!!).

Je me suis rasée la tête parce que les enseignants ont exigés de supprimer la lettre préparée par les étudiants, en menaçant d'un procès les représentants de ces derniers. Ils ont établi un climat de terreur et de chantage qui a démobilisé tous les étudiants présents. Je me suis rasé les cheveux parce que les enseignants ont comparé les étudiants aux nazis, en insinuant que les enseignants accusés étaient victimes de racisme et d'homophobie, une fois qu'un 
professeur est noir et l'autre gay. Il y avait une stratégie méchante pour l'inverser le scénario, me considérant comme l'agresseur, ainsi que tous les étudiants.

Je me suis rasée la tête parce que je suis fatiguée d'être harcelée, contrainte et subie au chantage. Je me suis rasée les cheveux parce que j'étais publiquement humiliée, offensée et manquée de respect par les enseignants qui ont dit que mes récits d'harcèlement sont du mensonge, sans même m'entendre personnellement et en détail. Je me suis rasée la tête parce que je souffrais d'un lynchage moral d'un Programme d'études supérieures auquel j'ai essayé de préserver à la fois leurs enseignants et les étudiants.

Je me suis rasée les cheveux parce que je pourrais me taire pendant sept jours et défendre ma thèse sans plus de contraintes et de coercitions, mais je me refuse de faire partie de cette logique oppressive que la faculté a lancé sur le Programme. Même avec la peur de représailles je ne me taise plus. Je me suis rasée la tête. Je me suis rasée les cheveux. Mais je suis restée de debout.

Le cas de Thais a eu des répercussions majeures sur les réseaux sociaux et les médias. L'étudiante a déposée une plainte contre l'enseignant et a réussi à être entendue par la Commission Parlementaire d'Enquête (CPI, en portugais), responsable pour enquêter les plaintes de violations des droits de l'homme dans les universités. Jusqu'au dernier jour de la collecte d'informations de notre recherche, il n'y avait toujours pas de date fixée pour la détermination de l'affaire.

Il convient de noter que le récit de soi en cherchant de l'engagement pour la vérité est aussi un travail éthique et politique. Toute «technique de soi» comprend, selon Foucault (2004), un mouvement actif de l'autoconstitution de la subjectivité, a partir des pratiques de la liberté. Ainsi, ceci est une technique de soi qui aspire la transformation politique de soi et des modalités de production d'énoncés qui créent une "relation entre l'existentiel et l'énonciation, entre l'affirmation de soi et la parole politique" (Lazzarato, 2014, p. 193).

Foucault (1995) souligne que de nouvelles formes de subjectivation émergent quand l'individu prend le risque pour exposer et expliquer les façons inhumaines qui fabriquent le sujet soumis, l'assujettissement. Cela exige du sujet le courage pour dire la vérité et révéler les mécanismes du pouvoir.

Si les relations de pouvoir pèsent sur moi quand je dis la vérité, et, si quand je la dit, j'exerce le pouvoir sur l'autre, alors je ne suis pas seulement en train de communiquer la vérité quand je dis la vérité. Je suis également dans l'exercice du pouvoir de la parole, en l'utilisant, en le distribuant, devenant le lieu de sa transmission et de sa réplication. (Butler, 2015, p. 159).

La parresia ${ }^{3}$ associée au récit de soi restructure et redéfinit le champ d'action possible, à la fois pour eux-mêmes et pour les autres, dans une dynamique d'autoconstitution existentielle et

3. Foucault $(1995,2004)$ considère en tant que techniques de soi les procédures par lesquelles un individu «s'approprie de soi», en devenant un sujet de ses propres pratiques et en construisant son soi dans une perspective éthique qui vise à se distancier des règlements et normativités l'Etat. Selon lui, ces techniques (méditation, écriture de soi, le régime, des exercices physiques et spirituels, la parresia ou le courage de la vérité) se rapportent à la capacité des individus de «faire pour eux-mêmes, un certain nombre d'opérations sur leurs corps, leurs âmes, leurs pensées et leurs comportements afin de produire une transformation en soi »(Foucault, 1984, p.785). 
politique : «Il y a une esthétique de l'énonciation, qui est ouverte à quelque chose de nouveau et laisse émerger la puissance du sujet et le sujet dans son potentiel" (Lazzarato, 2014, p. 199). En ce sens, nous pouvons dire que ces techniques de soi sont en mesure de promouvoir de nouvelles formes d'expériences et de subjectivités en établissant une autre scène énonciative, en nous retournant au monde comme des êtres transformés. La pratique politique de la parresia arrive quand un sujet prend le risque de dire la vérité, en exprimant ce que il pense vraiment et en se connectant à l'énoncé et à l'énonciation de façon à non seulement produire des effets sur les autres, mais à « affecter l'objet de énonciation, en produisant une transformation existentielle»(Lazzarato, 2014, p. 151).

Il nous semble que les appropriations des réseaux sociaux et de relations qui sont mises à jour par les sujets eux mêmes, constituent un espace qui permet, dans une certaine mesure, des utilisations créatives et subversives/ non soumises en tant que techniques de soi, car elles apparaissent comme «champ de possibilités d'action pour une multitude de comportements à être inventés (...) il comprend toujours des gestes, manières, modes, variations, résistances, bien qu'il sont apparemment minuscules ou inapparentes. » (Pelbart, 2013, p.232).

\section{LE COMMUN dE LA DOULEUR ET LA COMMUNAUTÉ DE VICTIMES}

Dans les exemples présentés ici, l'expérience épisodique de la douleur ou de la souffrance se présente comme une situation d'urgence superposée à tous les messages précédents qui ont alimenté le cours d'une vie commune et banale, dans la trivialité qu'elle était censé avoir. Dans ce lieu, la victime est présentée comme l'exemple réel d'une éventualité regrettable et inutile qui ne devrait pas se produire. Dans le groupe observé c'est l'expérience de la douleur provoquée par la violence sexuelle qui se somme aux nombreux cas du quotidien des jeunes femmes et des femmes adultes. Surtout, pour celles qui partagent leurs expériences de la douleur et de la souffrance et dénoncent la précarité et l'insécurité qui menacent constamment la vie et la dignité communes.

L'instance qui légitime la déclaration de vulnérabilité d'une telle victime ne se fait pas seulement pour la production d'un lien de témoignage, mais pour l'appel à la multiplication des voix, à la convocation à « rejoindre le chœur », à la participation qui se multiplie par le repost, par le retwitte, par le partage ou par les visualisations. Le succès (si on peut les considérer comme stratégie) de ces récits de soi analysés ici nous semble proportionnés à l'ampleur des partages et des visualisations possibles. Leur but est de produire des échos à la parole, à l'appel et à la fin produire un corps, constituer un visage, car c'est seulement ainsi qu'il est possible d'attirer l'attention publique sur ce qui ne devrait pas arriver.

Si dans les médias traditionnels les filtres éditoriaux, politiques, commerciaux, entre autres, cachent les faits afin d'offrir leurs discours préconçus, les dossiers et les écrits qui apparaissent et se multiplient dans les différents réseaux sociaux auraient tendance à être considérés comme des moyens de libre expression du sujet réel et ordinaire. Enfin, ce qui amène ces brèves récits au centre des discussions d'aujourd'hui est l'existence incontestable d'une certaine force motrice - si affective et réelle - qui ce fait par la conjonction des histoires de vie personnelles par les dispositifs technologiques de communication instantanée qui produisent, accumulent, partagent 
et se connectent instantanément autour de certaines situations vécues comme très intolérables et invisibles dans l'espace publique.

Ce changement provoque des variations et des déplacements importants des agents qui font partie de la narrative et qui mobilise à la fois les instances d'énonciation et de visibilité du moisujet dans un arrangement intime et particulière. Cette réciprocité entre les instances, de chaque partie, explique, tout d'abord que l'expérience narrativisée apparaît d'un peu détachée d'une position hiérarchique, traditionnellement contrôlée par les médias et les genres de communication médiatique, et traverse les différents domaines du quotidien de façon instantanée et plus directe. Deuxièmement, sa capacité pratique est mise à jour par un champ de plusieurs entraînements des sujets qui sont en relation. Par conséquent, la paire énonciation/visibilité est située et contextualisée par des matériaux expressifs qu'ensemble contractent de différentes manières, la relation entre le sujet, l'expérience et le moyen, en produisant d'autres réarrangements des scènes d'énonciation et d'interpellation par les différents types de déplacements interactifs et émotionnels.

Paulo Vaz (2010) établi une relation intéressante entre les souffrances (aujourd'hui considérées comme contingentes) et l'action humaine dans la culture contemporaine. Selon lui, le développement d'une société, mesuré grâce aux progrès technologiques, scientifique, sociale et politique, favorise une autre observation des souffrance quotidiennes, auparavant considérées comme nécessaires, mais qui, aujourd'hui, sont prises comme dépendantes de l'action humaine :

(...) tout d'abord, il faut tenir compte quelles sont les souffrances entendues comme contingentes, en étant à la portée de l'action humaine, et, d'autre part, quelle est la distribution des responsabilités entre l'action individuelle et l'action collective pour l'existence de ces souffrances sélectionnées (Vaz, 2010, p. 135).

L'auteur fait noter que l'apparition d'une souffrance contingente aujourd'hui ne se justifie que par la négligence et omission, notamment exercée par la politique de l'Etat, mais elle est encore délimitée par d'autres objectifs. D'où vient la corrélation immédiate de l'énonciation déclaratoire de la victime par rapport au fait, à la violence subie, présentée au public par la plainte et la convocation. Les récits-posts d'Amber et de Thais, parmi beaucoup d'autres victimes, réclament la place à la parole pour exhorter une responsabilité commune, parce que la déclaration d'une douleur expérimenté lie sa position de «victime inutile» à un coupable négligent et inefficace, pouvant, ce dernier, être représenté par l'état, la politique, la culture, la norme, entre autres.

Il est pas au hasard que ce type de récit-post se présente avec un lourd contenu déclarative d'accusation, parce que il ne suffit pas d'afficher les marques afin de supplier un regard complice et silencieux, mais de convoquer, en criant et hurlant, le partage de la douleur tel elle est subie et ses marques sur le corps lui-même. La production des récits de soi chez Amber ou Thais ne supplient pas le grief ou le confort de qui se soit, comme on le voit dans les cas de victimes de maladies, en semblent soumises sous le joug du destin et de la fatalité. La négligence et l'omission qui caractérisent l'attitude des institutions politiques responsables et de la société dans son ensemble, quand ils se gardent silencieux vis-à-vis de la violence dénoncée, est le point central à partir duquel se déroulent les cas violents et évitables qui cherchent à promouvoir le sens collectif d'indignation, de la commotion et, surtout, de la révolte de celui qui a le droit présumé de vivre la dignité de genre, religieuse, ethnique et humaine. 
Il est possible de noter que les profils des victimes qui se présentent sont directement liés à différentes formes de production et de mobilisation des récits de soi. Dans notre échantillon, le récit est produit par une attitude de réponse immédiate, c'est à dire, par la prise d'une position qui répond tout d'un coup, à l'urgence de la puissance déclarative de la douleur vécue et partagée des récits-posts.

Selon Valadier $(2011,2012)$ la vulnérabilité est une condition humaine qui peut toucher tout le monde à tout moment de la vie, raison pour laquelle elle se fait par le devoir d'être partagée. Chaque être humain est vulnérable parce qu'il est un être de chair, qui peut être affecté et touché dans sa sensibilité et corporéité radicale.

Butler (2011) affirme qu'on dépend des autres anonymes pour être considéré et reconnu. Dans cette dépendance serait notre condition de précarité et de vulnérabilité combinée. L'auteur développe l'argument selon lequel la précarité de la vie peut se manifester surtout dans la façon dont l'espace de l'apparence (souvent marqué par des cadres des médias) produit des formes différentes de distribution de la vulnérabilité, en provocant à certaines populations et groupes plus de risque à la violence que d'autres.

Dans l'argumentation de Butler, la représentation de l'altérité constitue un moyen d'humanisation / déshumanisation, de reconnaissance du lien éthique-morale avec l'Autre ou de justification de sa violation ou élimination. Ce qui nous lie moralement aurait rapport avec la façon dont le discours de l'Autre nous parle de telle sorte que nous ne pouvons pas l'éviter ou le détourner.

Ainsi, les récits de soit ont potentiellement le pouvoir de constituer un appel moral, un cri né de la souffrance qui est produite de façon commune, c'est à dire, que déclenche un moyen de construire ensemble une expérience de «l'écoute» des sons de la souffrance humaine et de la proximité avec la précarité de la vie.

\section{CONSIDÉRATIONS FINALES}

Les profils des victimes établis selon les récits de soi sur les réseaux sociaux analysés ici conjuguent des aspects politiques et moraux qui sont impliqués dans le processus de production / affichage / partage de la douleur et de la souffrance éprouvées. Raconter et se raconter sur les réseaux sociaux comprennent simultanément les conjugaisons du voir et de se voir, du rapporter un récit, du rapporter un récit de soi, ensemble.

Il est possible d'affirmer que les récits apportés dans les exemples de notre recherche se constituent comme technique de soi dans laquelle la parresia - le geste pour dire la vérité sans crainte, une vérité politique qui fait mal, provoque et déconstruit le status quo - a éclaté par les brèches, l'espace normalisé (de la parole et de l'image) des réseaux sociaux. La production du récit de ces jeunes femmes révèle l'intention claire de prendre le contrôle de leur propre vie, de devenir sujet de soi par l'entreprise de la réinvention de sa propre subjectivité, rendue possible par le récit de soi comme forme d'action éthique et politique.

Il s'agit de devenir auteurs de leur script pour affirmer un autre soi à la violation. Il s'agit également de chercher des éléments qui pointent vers une sorte de résistance aux formes vie prêtes, à l'effacement et la disparition de sujets dans les récits qui mettent les individus dans des cadres discursifs précédemment construits, en capturant ses gestes, routines, corps et paroles dans des 
opérations consensuelles, des contraintes et soumissions de toutes sortes. En ce sens, l'exposition et l'apparition du corps et du visage dans les récits de soi présentés ici sont capables de contracter des énoncés et des scènes d'énonciation qui révèlent une potentialité politique de désidentification, du dissensus et de la rupture.

Les victimes et leurs voix se déplacent dans ce dynamique de la vulnérabilité et de la douleur commune en cherchant la revendication de la parole pour faire entendre tous ceux qui sont évoqués et invoqués dans cette forme de partage.

\section{RÉFÉRENCES BIBLIOGRAPHIQUES}

Barros, V. A.; Silva, L. R. da. (2002). A pesquisa em história de vida. In I. B. Goulart (org.), Psicologia Organizacional e do Trabalho : teoria, pesquisa e temas correlatos (pp. 134-158). São Paulo : Casa do Psicólogo.

Bruno, F. (2013). Máquinas de ver, modos de ser. Porto Alegre : Sulina.

Butler, J. (2015). Relatar a si mesmo. Belo Horizonte : Autêntica.

Butler, J. (2011). Vida precária. Contemporânea - Revista de Sociologia da UFSCar, (1) : 13-33. São Carlos.

Fassin, D. \& Rechtman, R. (2011). L'empire du traumatisme. Enquête sur la condition de victime. Paris : Flammarion.

Foucault, M. (2004). A ética do cuidado de si como prática da liberdade. In. M. Foucault, Ética, sexualidade e política (pp. 264-287). Rio de Janeiro : Forense Universitária.

Foucault, M. (1995). O sujeito e o poder. In P. Rabinow \& H. Dreyfus (eds.), M. Foucault : uma trajetória filosófica para além do estruturalismo e da hermenêutica (pp.231-250). Rio de Janeiro : Forense Universitária.

Foucault, M. (1984). Les techniques de soi. In D. Defert, F. Ewald \& J. Lagrange (eds.), Dits et écrits. 1954-1988. Paris : Gallimard.

Goldemberg, M. (2004). A arte de pesquisar. Como fazer pesquisa qualitativa em ciências sociais. Rio de Janeiro : Record.

Hine, C. (2004). Etnografía virtual. Barcelona : Editorial UOC.

Hine, C. (2015). Ethnography for the Internet : embedded, embodied and everyday. London : Bloomsbury Academic.

Lazzarato, M. (2014). Signos, máquinas, subjetividades. São Paulo : Edições Senac, N-1 edições.

Pelbart, P. P. (2013). Subjetivação e dessubjetivação. In P. P. Pelbart, $O$ avesso do niilismo : cartografias do esgotamento (pp. 225-236). São Paulo : N-1 edições.

Rago, M. (2013). A aventura de contar-se : feminismos, escrita de si e invenções da subjetividade. Campinas : Editora Unicamp.

Recuero, R. ; Fragoso, S. \& Amaral, A. (2013). Métodos de pesquisa para internet. Porto Alegre : Sulina. 
Redação Marie Claire (2016, janeiro 7). Jovem ativista relata estupro nas redes sociais minutos após agressão. Revista Marie Claire. Globo.com G1. Disponível em : http://revistamarieclaire .globo.com/Web/noticia/2016/01/jovem-ativista-relata-estupro-nas-redes-sociais-minutos-ap os-agressao.html. Acesso em 09 fev. 2016.

Redação G1 (2015, março 6). Termina o prazo da comissão que apura denúncia de assédio na UFSCar. Globo G1. Disponível em : http://g1.globo.com/sp/sao-carlos-regiao/noticia/2015/03 /termina-o-prazo-de-sindicancia-que-apura-denuncia-de-assedio-na-ufscar.html. Acesso em 18 jan. 2016.

Sarti, C. (2011). A vítima como figura contemporânea. Caderno CRH, 24(61) : 51-61.

Sibilia, P. (2008). O Show do Eu : a intimidade como espetáculo. Rio de Janeiro : Nova Fronteira.

Staufenberg, J. (2016, janeiro 11). Instagram 'removes' post by activist Amber Amour 'live blogging' her rape. The Independent Journal. Disponível em : www.independent.co.uk/news/worl d/africa/instagram-removes-post-by-activist-amber-amour-live-blogging-her-rape-a6805491 .html. Acesso em 27 fev. 2016.

Valadier, P. (2011) Apologie de la vulnérabilité. Études, (414) : 199-210.

Vaz, P. (2010). A vida feliz das vítimas. In J. Freire Filho (org.), Ser Feliz hoje : reflexões sobre o imperativo da felicidade (pp. 135-164). Rio de Janeiro : FGV. 